\title{
PIWeCS: enhancing human/machine agency in an interactive composition system
}

\author{
IAN WHALLEY \\ The University of Waikato, Private Bag 3105, Hamilton, New Zealand \\ E-mail:musik@waikato.ac.nz
}

\begin{abstract}
This paper focuses on the infrastructure and aesthetic approach used in PIWeCS: a Public Space Interactive Web-based Composition System. The concern was to increase the sense of dialogue between human and machine agency in an interactive work by adapting Paine's (2002) notion of a conversational model of interaction as a 'complex system'. The machine implementation of PIWeCS is achieved through integrating intelligent agent programming with MAX/MSP. Human input is through a web infrastructure. The conversation is initiated and continued by participants through arrangements and composition based on short performed samples of traditional New Zealand Maori instruments. The system allows the extension of a composition through the electroacoustic manipulation of the source material.
\end{abstract}

\section{INTRODUCTION}

Research towards creating the PIWeCS (Public Space Interactive Web-based Composition System) infrastructure began from the need to solve an artistic problem, rather than extend technology. In New Zealand, there is increasing public interest in traditional Maori instruments, partly resulting from the work of Richard Nunns and the late Hirini Melbourne (Melbourne and Nunns 1996). Recently, New Zealand composers have also been interested in extending these instruments in new artistic contexts.

Access to leading players of the instruments is limited, and many of the instruments are unique. Given these restrictions, the initial intentions of the PIWeCS project were: to find ways to overcome barriers of time and scarcity to allow lay people to discover and explore some of the instrumental sounds in a public space, independent of passive reception through live performance and the CD medium; to allow them to explore the combination of some of these instruments in a compositional and interactive context; and to allow the possibility of extending the instruments in an electroacoustic context as a way of exploring a new hybrid sonic landscape unique to New Zealand.

Implementing a system meant first reviewing web-based and interactive systems and assessing their strengths and weaknesses according to the needs of the project. From this, two limitations in machine/human systems needed to be addressed in PIWeCS: the lack of interactivity in many so-called interactive installation systems; and the lack of a means to implement an intelligent bi-directional dialogue between machine/human agency.

Evolutionary systems were examined as a possible medium to address a greater sense of interactivity, but were found to be comparatively unsuited to dealing with the problem in context. The solution adopted for PIWeCS resulted in the integration of an intelligent agent system to enhance machine agency, coupled with MAX/MSP, allowing groups of people to take an extended Shaper Approach (Weinberg 2002) to the source material though a web interface.

The PIWeCS infrastructure addresses many of the limitations of the web-based systems surveyed for its purposes, and implements a complex composition system based on a dialogue between human/nonhuman agency as a way of exploring the sampled material. The approach differs from Paine's (2002) proposed implementation of his conversational model based on streamed data and dynamic morphology in that PIWeCS incorporates a reactive system, is based on traditional instrument samples, and takes a modular approach in integrating an interactive component.

The focus of this discussion is on the process of research, aesthetic considerations, and the resulting infrastructure of PIWeCS as human/machine agency.

\section{WEB-BASED SYSTEMS}

The PIWeCS system needed to be mounted in a public space as a primary means of interaction. It was also envisaged that it might involve simultaneous participation in the public display from off site, so Internetbased composition systems were a starting point in the search for a suitable technical infrastructure. Recent surveys of Internet systems for music-making include Weinberg (2002) and Ballet and Wohrmann (2002). From an aesthetic perspective, Weinberg (2002: 352-4) outlines four approaches based on different levels of interconnectivity through the Internet.

The Server Approach allows participants to communicate with a central server, but not with other people interacting with the system. An advantage is that each individual's experience can be unique, and 
each user can be involved in creating the music because participants may contribute their own sequences. The approach assumes a degree of musical literacy in order to participate. The Bridge Approach lets the network act as an intermediary between real performers, so performers might improvise as if they were in the same physical space. The network simply provides a means for participation, listening and response.

The Shaper Approach uses the network's central system in an active role by generating material algorithmically, and people may shape and modify it. They can listen and respond to the collective input of others, but there is no direct communication between participants. The Construction Kit Approach allows experienced musicians to contribute their own material and alter others' contributions. The musical structure is usually set and not influenced by the dynamic input of participants. Users only refresh the content.

Selecting a suitable framework for PIWeCS was driven by the intention of the project: to encourage interaction and exploration of the samples of traditional Maori instruments in a compositional setting, as well as their electroacoustic extension. The potential audience/participants for the project may vary from children, to interested lay people, to experienced musicians. The system might also be implemented in more than one space simultaneously.

Two issues were immediately apparent. First, the less musical experience participants had, the more active the system might have to be to encourage engagement, but it would also need to be accommodating enough to allow extension for experienced musicians. Thus the system would need to be flexible to cope with a range of inputs. Secondly, a decision had to be made as to the aural output; was it going to be an individual one, or to be a collective one that amalgamated the efforts of many players.

A decision was made to start from a Shaper Approach; the resulting work would then be a collective effort. This was necessary because of the public nature of the project. The approach would be extended in a public context, however, because it was hoped that people on site would interact with each other independently of machine input as the work unfolded. As the focus of the project was educational, this interaction was seen as adding to the project.

However, at times there would be people interested in the project that may not be able to attend the public space 'performance', and they needed to be included in some way. For the sake of consistency, the same mode of machine interaction needed to be available to both groups of participants: primarily a web client interface for interaction and feedback.

Given the possible combinations of on- and off-site participation, it was decided to limit the number of on-line connections and have the main form of interaction take place in the public space. This meant that the work was primarily driven from the public space as a collective product. Limiting off-site access was also partly necessary to protect the copyright of the source material and resulting compositions.

The accepted disadvantage of the Shaper Approach as a starting point to infrastructure was the loss of the individual experience provided by the Server Approach. This compromise seemed worthwhile, however, given that the source material would be unfamiliar to many people, and dialogue between participants on site may aid its exploration.

The technical infrastructure necessary to implement composition systems over the web in combination with MAX/MSP is increasingly widespread (see, for example, Hajdu 2003). The immediate problem in comparison to Hajdu's system - one based on connecting live performers (a Bridge Approach) - is that the Shaper Approach connects humans to machine, and the machine is largely reactive if based on MAX/ MSP and sample triggering. It was therefore decided to explore ways to enhance the machine agency as a way of increasing the sense of human/machine interaction, and allow greater compositional exploration of the source material.

\section{INTERACTIVE SYSTEMS}

Given the nature of the PIWeCS source material and possible skill range of participants, it seemed useful if the machine as agent could be made to 'share rather than imitate': if the machine could take the role of an intelligent listener and participant in a dialectic, rather than respond with a set of conditions regardless of the situation, or generate a series of patterns regardless of exploration paths and directions users might explore.

In recent literature on interactivity, Paine (2002) argues that a significant problem with many machine based 'interactive' works is that they react rather than interact, and in line with the work of Bongers (2002) concludes that many interactive systems lack a sense of cognition and dynamic intelligence to claim an interactive title.

Paine (2002: 297) suggests that to address the emerging possibilities of the interactive idiom, a new approach is needed between human/machine agency he terms the conversational model. Based on the analogy of a human conversation, he notes that this relationship should be unique and personal to participants, unique to the moment of interaction, vary with unfolding dialogue, and be maintained by both parties speaking the same language and addressing the same topic. Further, one party may know the beginning point of a conversation, and while there may be a preexisting agenda, the terrain of the conversation might be unknown in advance. The process is then one of 
exchange and of sharing ideas, and the relationship between participants should deepen over time.

Paine advocates that the medium best suited to implement and understand the new approach is found in public-based installations. Internet lattice-based (pitch and time) models, or those that are instrumentally driven are described as inappropriate to take advantage of the emerging possibilities. This is because they include pre-existing musical frameworks, which he describes as only making sense in interactive music systems that are designed for accompaniment or improvisations that adhere to the same prescription. Similarly Paine (ibid.: 301 ) suggests that the composer should not create all the resources needed for a work before it is realised, because this limits the outcomes that might evolve as the result of dynamic interaction.

The aesthetic/technical solution Paine (ibid.: 304) proposes involves 'the creation of sensing systems that explore streams of input rather than individually triggered events'. His solution is intended to be implemented using object-oriented programming, and expressed though Wishart's (1996) notion of dynamic morphology in sound, rather than traditional (Western) instrumentation and notions of pitch duration. Smalley's (1996) aesthetic approach underpins this solution.

For PIWeCS, using the conversational model to increase a sense of interaction seemed a worthy starting point, but Paine's solution to implement it was inappropriate. First, the source material was instrumental, although not lattice based in a Western music sense. Second, performance gestures, many of which were unique to the instruments played, were captured in sampling the material to be manipulated, and the combination of this material through triggering it in new combinations was of interest. Further, the unique nature of the instruments meant that their electroacoustic exploration through sound processing devices might result in outputs that were not imagined by the system designer. Finally, the nature of the conversation may be very asymmetrical given the source material and varying levels of knowledge and ability that participants brought to the situation.

An alternative to Paine's suggested implementation then needed to be found for enhancing human/ non-human interaction. The solution also needed to be realised within the shaper/exploratory approach that suited the PIWeCS aesthetic. Aspects of 'cognition' would then need to be added to the machine agency. The metaphor was to think about the machine as a type of benevolent composition aid, but with a unique output that would evolve in the dialogue with participants in the same way a group of musicians might improvise the content and structure of a new work with a group of amateurs playing in an unfamiliar idiom.

\section{ADDING MACHINE COGNITION}

The idea of a smart machine that could hold an intelligent conversation may be science fiction, but the areas of artificial intelligence (Miranda 2000, 2001), evolutionary systems and intelligent agents held out the promise of implementing some small aspects of a conversational model for the needs of PIWeCS. Two possible approaches were examined.

At first blush, evolutionary systems techniques seemed a way to implement aspects of machine 'cognition'. Recent surveys given by Brown (2001) and Bentley and Corne (2001) give a broad overview of the field and its application to music and art. Arcay (2000) outlines how the technology has been used as an optimisation technique in creating tonal music with a combination of human input as critic, expert systems as input, and neural network learning. Bentley and Una-May (2001) note how creative evolutionary systems have grown away from their beginnings as optimisers and into the field of generative systems to assist creativity. Dorin (2001) describes the broad area of generative systems in the arts. The evolutionary approach is widely used in other arts, such as graphic design (see Corlabella and Soddu 2003).

Despite the range of work in the field, the focus of this technology on optimisation and creative/ generative assistance appeared unsuited to implementing a conversational model in real time, and was abandoned after an initial exploration of the tools and techniques. This was partly because more effective tools to address most of the problems were available in the emerging field of intelligent agent systems.

An agent is someone or something that acts on your behalf. Software-based agents may exhibit varying degrees of persistence, independence, communication and collaboration with other agents or humans. 'Intelligent' agents (see Bigus and Bigus 2001) might include decision-making capabilities, the capacity to learn in an environment, and mobility over networks. The scope of an agent's ability is related to their level of independence. More 'intelligent' agents monitor environments, glean information, make a decision to react or not, and modify their behaviour according to the results received. Agent systems can be implemented in software packages such as Aglets, Grasshopper, Jade and Zeus (see http://www.agentbuilder.com/ AgentTools/index.html)

Recent applications of agent technology to music include attempts to capture the 'artificial performer' in software when applied to tonal music (Nakayama 2003), or to manage complexity in real-time performance systems that require human input (Spicer, Tan and Tan 2003). Mobile agents are used in the Andante system (Kon and Ueda 2003), where the creation and performance of music can be realised within networkdistributed environments using synthesis. Chen and Kiss (2003: 11) recently displayed an interactive tool 
that 'makes use of a multi-agent system composed of an environment generated by synthesised sounds and images and containing a number of objects that may be perceived, created, destroyed and modified by the agents'.

Conceptually, intelligent agent technology is a closer 'fit' to the requirements of a conversational model than evolutionary systems. However, of the recent work found that applied the technology to music, none integrated it with existing 'reactive' software packages, used sample manipulation systems, or an Internet interface. Similarly, current work had not addressed enhancing interactive dialogue based on human/machine agency as a complex system, the focus being mainly on self-referential systems or enhancing human control over machines. Recent applications of agents to music did, however, point to the potential of the technology to integrate a degree of cognition into the Shaper Approach to the source material on which PIWeCS had settled.

\section{PIWECS APPROACH}

Given the educative requirements of PIWeCS and the above survey of appropriate methods to implement it, the broad technical requirements of the system were then as follows.

(1) There was a need to separate the interface from the 'instrument', because the instruments were largely created, and were only to be manipulated through the Internet. Barbosa (2003) outlines the merits of dividing machine from controller in distributed systems, allowing for the most suited technology and programming languages to be used in a modular approach to building systems to enhance their flexibility.

(2) There would need to be interfaces that were common to public and private users, and familiar and comfortable input devices would need to be selected so there was little need to 'learn the instrument'.

(3) The machine aspect of the system must be capable of engaging in a conversation and establishing a dialogue, however limited, to enhance this engagement. It must be able to listen, initiate, suggest, react, follow, and comment on human input. Further, the machine contribution must be unique to each occasion and learn something about the preferences of participants interacting on a specific occasion.

(4) The initial mapping of web interface input gestures to acoustic sample responses must aim to be simple, clear and immediate; and the aural and visual feedback to participants should correspond to inputs in such a way as to make their input to the system recognisable.

(5) Mappings that might involve the manipulation of the material in electroacoustic idioms, either in isolation or in combination with the acoustic source material, should aim to have a visual equivalent that was clear and accessible to the range of participants taking part in the work.

(6) The system would have to work in a physical space like an art gallery, but also online for off-site participants, and be suited to combining both points of access.

(7) The web interface on the client side needed to be flexible so that it could be made graphically interesting but also relevant to allow sound manipulation.

\section{TOOLS AND INFRASTUCTURE}

Using a modular approach to infrastructure meant that there were many software packages available to build the PIWeCS system. Further, projects in the literature had partly implemented aspects of the system. This saved building a system from scratch.

To create a simple reactive sample-based mechanism, the ubiquitous MAX/MSP was used. Electroacoustic manipulations of this material in the patch were made possible through the use of some purposedesigned modules and specifically designed packages such as Pluggo.

Joining the MAX/MSP patch based on a serverto-web-client interface began from Young's (2001) work. Young implemented his system by using Flash on the client side to trigger MAX/MSP on a server. His approach relied on using Flash's built-in method of addressing Javascript that passed data to a purposedesigned 'Teleapplet'. The process, known as LiveConnect, was supported by most web browsers. The data was then passed to MAX by using Open Sound Control (OSC) developed at UC Berkeley Centre for New Music and Audio Technologies (CNMAT).

A limitation of this approach was the complicated data path, and it would not work on the Macintosh OS9 operating system with Internet Explorer. However, recent software updates to Flash and OSC have addressed these issues and allowed improvement in the approach. Flash $M X$ allows for XML data to be sent from a web-based client to a server. OSC developments (Freed 2003) have since included the implementation of FLOSC, allowing OSC to read XML and communicate with MAX. PIWeCS adopted this updated Flash/FLOSC communication format.

A web interface and mouse provided the main controllers for participants through on- and off-site computer terminals. This was based on a mixing board concept, with additional buttons for each channel to trigger events and effects. To enhance interaction, mobile phone triggering was investigated as a further method of engagement. The decision was partly based on ad hoc observations of the adept use of these devices by their many users, particularly young 
people, and their familiarity with their telephones ('instruments') as data transmission/reception devices.

Initial experiments with WAP (Wireless Application Protocol) as a transmission medium proved it was too slow to be of use in the PIWeCS system. However, text messaging was faster, more familiar to phone users as an interactive device, and easier to integrate into the system. A similar approach to integrating mobile phones as the control device to interact with public sound installations has been used recently in The Intelligent Street Project (see http://www.intelligentstreet. net/). A limitation when compared to the computerbased web interface is that interface parameters are much more difficult to manipulate or control quickly.

Many software platforms were investigated for the implementation of the intelligent agent aspect of the infrastructure (see Bigus and Bigus 2001). The differing foci of these packages makes them more suitable to developing applications such as mobile agent systems, network distributed decision systems, or multi-agent systems on single machines. A multi-agent system (MAS) approach was adopted, as implemented in packages such as Agentbuilder and Jade.

The block technical architecture of the PIWeCS is given in figure 1. A further advantage of separating the control mechanism from the 'instrument' meant that the mechanism for dialogue between human and machine agency, and the conversation model, could be implemented in a computationally efficient way in real time in the digital domain.

Technically, XML data is streamed from Flash and read by a purpose-developed multi-agent system (MAS) for PIWeCS. The MAS, depending on the requirements at any given moment, could pass the data directly to MAX/MSP, or alter it after gathering information on users' input. It then sends the data on to the MAX/MSP patch. Information on decisions made by humans and the MAS could be sent back to participants' browsers from MAX/MSP, providing visual feedback on the combined input into the system.
Audio information was sent back to participants through the Internet using Internet audio streaming methods, also adopted in systems like Young's (2001) and Hajdu's (2003). Mobile phone users then needed to be at a public performance to receive the audio information, since it was anticipated that they would interact with the system at a public exhibition that had audio playback.

\section{CONTENT, CONVERSATION AND AESTHETIC}

Gathering the samples for the system involved recording short motives of traditional Maori instruments played by Richard Nunns, one the foremost players in this idiom. The samples were classified into acoustic groups such as percussion instruments, drones, tunes, and effects according to the way Nunns understood and had previously used them in a musical context (Melbourne and Nunns 1996). The samples were then loaded into a MAX/MSP patch, and the patch programmed to receive instructions for their arrangement and manipulation from human or machine agency.

On a uni-directional level, using Young's (2001) approach to infrastructure and Winberg's (2002) notion of a Shaper Approach, one could allow users to quickly shape the samples into an arrangement using the web interface. For example, different parts of the source material could be combined, looped and fragmented by the users.

Users may also develop a sense of a 'composition' by manipulating simple global parameters such as unity/variety, volume or tempo. They could also alter individual instruments through compositional methods such as pan placement, and add effects to instruments. This allowed users to give a sense of structure to the material, and develop material as they saw fit. Aesthetically, the acoustic manipulation

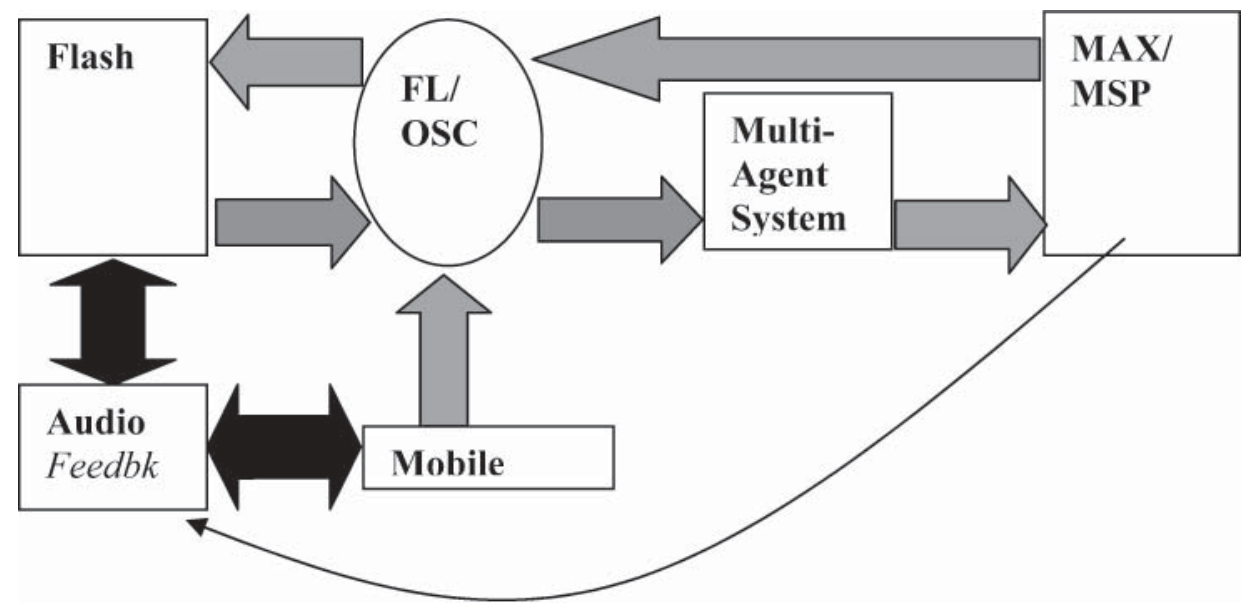

Figure 1. Data stream and audio feedback. 
possibilities coded in MAX/MSP were based on the way the instruments were used in previous work by Nunns as a starting point. Using this approach assisted lay people in initially exploring the source material.

Up to this point, the system is still 'reactive' according to Paine's (2002) definition, and there is little sense of the machine agency entering into a dialogue in any cognitive sense. By adding a MAS to the chain of control (figure 1), a sense of machine 'intelligence' is introduced as an aspect of a 'complex system' to enhance machine/human agency.

The MAS contains a number of components. A reception agent receives (listens to) human users' input, or notes a lack of this input, collecting data sent by users from the interfaces. From this data, the reception agent can make an initial assessment about the level of skill a group of users has, by comparing their input to a range of possible manipulations that can be made by the MAS to the samples embedded in the MAX/MAP patch. The reception agent can then decide on the level of influence the MAS will have on the machine dialogue with human agency.

In this sense, the MAS acts as an expert system in the first instance. If the decision is made for the machine agent to join in the dialogue through MAS, a helper agent aids with arranging and developing material if the human participant's skill level is low. An extender agent allows a range of electroacoustic transformations of the material to be introduced through using audio effects.

Allowing the MAS greater flexibility than a simple rigid expert system is an unsupervised learner agent (Bigus and Bigus 2001: 151). This allows for the data streamed from the users to be understood in terms of recurring patterns and paths that are unique to each group. This information can be used to influence the role of the helper agent and the extender agent in the MAS. The approach allows a discourse to occur between modules in the MAS.

Through using the MAS as part of the technical infrastructure, a sense of a conversation between humans and machine agent can then be developed. The process begins from scripting the MAS with a range of compositional possibilities to trigger a MAX/ MSP patch, and allowing the MAS to listen, adapt, respond and suggest outputs to groups of users according to their actions. The MAS can lead, follow or combine these approaches at different points of the process. A dialogue can then unfold beginning from a common topic, even if the initial language (sample sources) is unfamiliar to many users. More importantly, conversation between a group of people and machine agency can develop in unique ways, and the relationship between human and machine agency can deepen over time, although within the accepted limitations of the ma:Shaper Approach to web-based music interaction adopted (see above).
A high level of expertise is scripted into the MAS based on the author's knowledge and experience of the instruments and their electroacoustic manipulations (Whalley 2002). This was partly necessary because it was expected that the low skill level of many people wanting to interact with the system might result in a lack of interesting sonic outputs and therefore discourage engagement. In this sense, with a conversational modus operandi, the MAS becomes a 'partner' in the composition process, but takes the lead in an adult/ child paradigm if needed. Rather than undermine the conversational aesthetic, it accepts that many conversations are based on asymmetrical relationship of knowledge and reinforces the educative and exploratory intent of the system.

At the risk of imposing an aesthetic on the source material, the view was that exploring the material using a conversational modus operandi was a part of the wider exploration of traditional Maori instruments also happening in other idioms, such as Nunns' recent performances with the New Zealand String Quartet, and his work with jazz practitioners such as Mike Nock. In the electoacoustic field, recent work also combines fragments from Richard Nunns' performances with electroacoustic idioms.

PIWeCS then permits the combination of composer and performer in the digital realm to allow lay people to interact with the encoded expertise, but in a way that participants may evolve an independent level of expertise as part of the engagement process through an evolving dialogue.

With the range of participants that may use the system, it was hoped that through the conversational approach implemented, new sonic directions never initially envisaged by the contributing composer and performer might be explored. In this way, ideas may be exchanged between human and machine agency, but the direction of the conversation and the ground it may cover are unknown in advance.

\section{MAPPING}

Mapping gestures in electroacoustic music, musicbased installations, and new instrument design is of course problematic and often contentious (Hunt 2002).

As the samples for PIWeCS were recorded as performance fragments rather than built as instruments to be played, initial mappings between user and system were comparatively simple. At a musical arrangement level, the users were just turning different combinations of performance gestures on or off through a mixing board interface. In this sense, a performance element and mappings were embedded in the resulting composition at a sample level.

An advantage of the source material was that it did not follow a synchronous beat, and many of the samples have little sense of steady pulse. Further, the 
Western notion of diatonic harmony is not central in traditional Maori instrumental music. This allowed for a far greater flexibility in combining instruments than would be possible in situations requiring Western diatonic tonal harmony. Similarly, issues arising from network latency, critical in much tonally based rhythmic music, were then less problematic.

The ability to author a new work, in the sense of giving it shape and direction, requires compositional skill. A series of menus mapped to basic structural parameters within the Flash interface are useful for a knowledgeable musician, but often confusing to a novice. Improving the graphic interface to map mouse gestures into controlling compositional elements is an ongoing task.

Visual mapping becomes more problematic when users go into the electroacoustic effects processing area. Generally, while many lay people are familiar with simple concepts such as pan, the use of many basic electroacoustic devices such as dynamic filtering are often confusing without visual feedback. This is particularly evident when obscure plug-ins are used with names that have little relationship to the effect heard. The mapping of the electroacoustic transformations into feedback to the visual interface started from the principle of 'what you hear is what you see'. This is relatively simple to implement with devices like volume or turning an effects button on or off, but is an area that becomes problematic with more complex audio effects transformations and the sheer number of possible combinations that need to be represented on screen. The PIWeCS system as a prototype is an evolving one, and work is continuing in areas such as the efficient and relevant representation of input and output on the web interface screen.

\section{SCOPE AND DIRECTIONS}

Paine's (2002) conversational model provided one of the conceptual starting points for this discussion, but his suggested implementation of the model and the broad aesthetic on which this is based significantly varies from the PIWeCS system. Paine (ibid.: 301) suggests that mainstream approaches to interactive systems, where the scope is set by the composer/ programmer at the outset or based on a set group of instruments with an inherently limited aesthetic and morphological scope, constitute a 'limitation that in my view has no place in interactive electronic music performance'. His criticism is largely of existing systems based on Western tonal instrumental music models (Winkler 1998). His proposed solution is based on an innovative aesthetic preference (Wishart 2002) that seems partly a matter of choice and intent.

PIWeCS uses human/machine agency to create compositional explorations of pre-existing material. The intention is educative, and the aesthetic communicative and creative. The integration of samples with multi-agent technology implements a different approach to the conversational model. The source material is non-Western and instrumental. The aesthetic limitations when judged against Smalley's (1997) innovative view of acousmatic art based on non-instrumental gestures are accepted. In contrast, the ability to create within and extend known archetypes reflects a view where the needs of innovation and communication are more carefully balanced in a creative outcome (see Milicevic 1998; Whalley 2000).

A limitation of the PIWeCS system, that Paine (2002: 301) suggests is typical of many conventional synthesis-based interactive systems, is that it does not allow for the creation of new algorithms as part of the dialogue between user and creator. The assumption is that users will bring a level of music/sound expertise to the situation, and the machine/human agency dialogue is symmetrical. The trade-off in the PIWECS implementation of the conversational model is a structure to accommodate differing levels of knowledge, skill and information people bring to the situation.

An area remaining to be explored is real-time or static visual representation of the instruments, showing how they are played. The look of an instrument and knowing how sound is produced may help (but may also hinder) how people interact with the system. Although video material was collected along with the sound samples, and a binary correspondence would be possible using the program Jitter to add short visual clips to PIWeCS users' selections, the visual referencing becomes problematic with compositional manipulation such as digitally transposing an instrumental sample into a different sonic register. Further, mapping a picture/audio correspondence becomes challenging as the aural material is manipulated electroacoustically.

\section{ACKNLOWLEDGEMENTS}

My thanks are due to Ross Peart for aid in Java programming to integrate the agent software; and to Waikato University for funding aspects of the research process. The project would not be possible without the unique traditional Maori instruments and performance expertise of Richard Nunns. His generous support is gratefully acknowledged.

\section{REFERENCES}

Arcay, B., Dorado, J., Rodriguez, J., Romero, J., and Santos, A. 2000. Evolutionary computation system for musical composition. In Mathematics and Computers in Modern Science, pp. 97-102. World Scientific and Engineering Society Press.

Ballet, G., and Wohrmann, R. 2002. Design and architecture of Distributed Sound Processing Systems for web-based computer music applications. Computer Music Journal 23: 73-84. 
Barbosa, A., Kaltehbrunner, M., and Geiger, G. 2003. Interface decoupled applications for geographically displaced collaboration in music. Proc. of the Int. Computer Music Conf., pp. 199-202. Singapore.

Bentley, P., and Corne, D. 2001. Creative Evolutionary Systems. Morgan and Kaufmann.

Bentley, P., and O'Reilly, U.-M. 2001. Ten steps to make a perfect creative evolutionary design system. In Proc. of GECCO 2001 Workshop on Non-Routine Design with Evolutionary Systems. Sydney University, http://www. cs.usyd.edu.au/ josiah/gecco2001_workshop_schedule. html

Bigus, J. P., and Bigus, J. 2001. Constructing Intelligent Agents in Java (Second Edition). John Wiley \& Sons.

Bongers, B. 2000. Trends in gestural control of music. In Physical Interfaces in the Electronic Arts. Interaction Theory and Interfacing Techniques for Real-time Performance. IRCAM - Centre Pompidou.

Brown, A. 2002. Opportunities for evolutionary music composition. Proc. of Australasian Computer Music Association Conf., pp. 27-34. Melbourne.

Chen, C., and Kiss, J. 2003. Setting up a self-organized multi-agent system for the creation of sound and visual virtual environments within the framework of a collective interactivity. Proc. of the Int. Computer Music Conf., pp. 11-14. Singapore.

Corlabella, E., and Soddu, C. 2003. Generative Art, Predludi, Natural Mirrors. 11 th Proc. of the Int. Symp. on Electronic Arts, pp. 52-3. Nagoya, Japan.

Dorin, A. 2001. Generative processes and the electronic arts. Organised Sound 6(1): 47-53.

Freed, A., Wright, M., and Momeni, A. 2003. Open sound control: State of the Art 2003. MINE-03. Int. Conf. on New Interfaces in Musical Expression, pp. 153-9. Montreal, Canada.

Hajdu, G. 2003. Quintet.net: A quintet on the Internet. Proc. of the Int. Computer Music Conf., pp. 315-18. Singapore.

Hunt, A., Kirk, R., and Wanderley, M. 2000. Towards a model for instrumental mapping in expert musical interaction. Proc. of the Int. Computer Music Conf., pp. 202-11. Berlin.
Kon, F., and Ueda, L. 2003. Andante: a mobile musical agents infrastructure. Proc. of the IX Brazilian Symp. on Computer Music, pp. 87-94. Campinas, Brazil.

Melbourne, H., and Nunns, R. 1996. CD. Те Ku Te Whe. Rattle, RAT D004.

Milicevic, M. 1998. Deconstructing musical structure. Organised Sound 3(1): 27-34.

Miranda, E. (ed.) 2000. Readings in Music and Artificial Intelligence. Harwood Academic Publishers.

Miranda, E. 2001. Composing Music with Computers. Focal Press.

Muller, S., and Schubiger-Banz, S. 2003. Soundium2: an interactive multimedia playground. Proc. of the Int. Computer Music Conf., pp. 301-4. Singapore.

Nakayama, L., Wulfhorst, R., and Vicari, R. 2003. A multiagent approach for musical interactive system. Proc. of $A A M A S^{\prime} 03$, pp. 584-91. Australia.

Paine, G. 2002. Interactivity: Where to from here? Organised Sound 7(3): 295-304.

Smalley, D. 1997. Spectromorphology: explaining soundshapes. Organised Sound 2(2): 107-26.

Spicer, M., Tan, B. T. G., and Tan, C. 2003. The learning agent based interactive performance system. Proc. of the Int. Computer Music Association Conf., pp. 95-9. Singapore.

Warde, A. 1999. Change over Time: responsibility and power in the midst of catastrophe. Leonardo Music Journal 9: 95-101.

Weinberg, G. 2002. The aesthetics, history, and future challenges of interconnected music networks. Proc. of the Int. Computer Music Association Conf., pp. 349-56. Göteborg, Sweden.

Whalley, I. 2001. Systems dynamics modelling: applications to computer music. Organised Sound 5(3): 149-57.

Whalley, I. 2002. Kasumi. New Zealand Sonic Art III. University of Waikato, UWMD 1203: Track 5.

Winkler, T. 1998. Composing Interactive Music, Techniques and Ideas Using Max. MA: The MIT Press

Wishart, T. (ed. S. Emmerson) 2002. On Sonic Art. Routledge.

Young, J. P. 2001. Using the web for live interactive music. Organised Sound 6(2): 107-10. 